\title{
Percutaneous coronary intervention versus coronary artery bypass grafting in heart transplant recipients with coronary allograft vasculopathy: a systematic review and meta-analysis of 1,520 patients
}

\author{
Jessica G. Y. Luc ${ }^{1}$, Jae Hwan Choi ${ }^{2}$, Syed-Saif Abbas Rizvi², Kevin Phan ${ }^{3}$, Ester Moncho Escrivà ${ }^{4}$, Sinal \\ Patel $^{2}$, Gordon R. Reeves ${ }^{5}$, Andrew J. Boyle ${ }^{5}$, John W. Entwistle ${ }^{2}$, Rohinton J. Morris ${ }^{2}$, H. Todd Massey ${ }^{2}$, \\ Vakhtang Tchantchaleishvili ${ }^{2}$ \\ ${ }^{1}$ Faculty of Medicine and Dentistry, University of Alberta, Edmonton, Alberta, Canada; ${ }^{2}$ Division of Cardiac Surgery, Thomas Jefferson University, \\ Philadelphia, Pennsylvania, USA; ${ }^{3}$ Faculty of Medicine, University of New South Wales, Sydney, Australia; ${ }^{4}$ Department of Statistics, University of \\ Granada, Granada, Spain; ${ }^{5}$ Division of Cardiology, Thomas Jefferson University, Philadelphia, Pennsylvania, USA \\ Correspondence to: Vakhtang Tchantchaleishvili, MD. Assistant Professor of Surgery, Division of Cardiothoracic Surgery, Thomas Jefferson University, \\ 1025 Walnut St, Suite 607, Philadelphia, PA 19107, USA. Email: Vakhtang.Tchantchaleishvili@jefferson.edu.
}

Background: Transplant coronary artery vasculopathy (TCAV) is the major cause of late allograft failure and death in heart transplant recipients. The aim of this systematic review was to examine the outcomes of percutaneous coronary interventions (PCIs) as compared to coronary artery bypass grafting (CABG) surgery in the management of TCAV. Our secondary objective was to compare the use and outcomes of drug eluting stents (DES) as compared to bare metal stents (BMS) in this patient population.

Methods: Electronic search was performed to identify all studies in the English literature examining PCI as compared to CABG for TCAV in heart transplant recipients. All identified articles were systematically assessed for inclusion and exclusion criteria.

Results: Of the 4,989 studies identified, 29 studies were included. Among 1,520 patients who developed TCAV, 1,470 patients underwent PCI and 50 patients underwent CABG. There were no significant differences in baseline demographics and comorbidities among the PCI and CABG cohorts. Compared to the PCI cohort, patients who underwent CABG had a higher early mortality (CABG 36.4\% vs. PCI 4.3\%, $\mathrm{P}<0.001$ ) and overall mortality (CABG $42.3 \%$ vs. PCI 21.4\%, $\mathrm{P}=0.049)$. When comparing DES versus BMS cohorts, there were no significant differences in the rate of in-stent stenosis (DES 14.5\% vs. BMS 24.4\%, $\mathrm{P}=0.476$ ), overall mortality (DES $17.4 \%$ vs. BMS 30.8\%, $\mathrm{P}=0.302$ ) or cardiac related mortality (DES $7.7 \%$ vs. BMS $21.8 \%, \mathrm{P}=0.415)$.

Conclusions: CABG and PCI are both feasible modalities for revascularization in patients with TCAV where PCI is associated with lower mortality. There were no differences in outcomes among patients who underwent PCI with DES as compared to BMS. Potential bias may exist due to heterogeneity in available data. Further studies are needed to delineate evidence-based guidelines to tailor the appropriate therapy, CABG or PCI, to the appropriate patient.

Keywords: Percutaneous coronary interventions (PCIs); coronary artery bypass grafting (CABG); revascularization; heart transplantation; transplant coronary allograft vasculopathy

Submitted Dec 06, 2017. Accepted for publication Jan 06, 2018.

doi: 10.21037/acs.2018.01.10

View this article at: http://dx.doi.org/10.21037/acs.2018.01.10 


\section{Introduction}

Cardiac transplantation is the definitive therapy for patients with end stage heart failure refractory to medical management $(1,2)$. Transplant coronary artery vasculopathy (TCAV) remains the most common cause of late allograft failure and death after 1 year (3). The prevalence of TCAV has been reported to be $8 \%, 30 \%$ and $50 \%$ at 1,5 and 10 years post heart transplantation, respectively (3).

In contrast to coronary artery disease (CAD) that is associated with focal atherosclerotic calcium plaque formation, TCAV is a panarterial disease in which there is diffuse vessel narrowing due to concentric fibroproliferative lesion of the intima without calcium deposition (4). In addition to morphological differences, a different set of risk factors has also been suggested in the development of TCAV as compared to CAD. The pathophysiology of TCAV is multifactorial and involves both immunological and nonimmunological mechanism. Immunological factors that have been suggested include HLA mismatching, T cell activation, cytomegalovirus infection, older donor, younger recipient and presence of recipient pretransplant cardiovascular risk factors (5-7). Nonimmunological factors include endothelial injury, ischemia-reperfusion injury and risk factors for CAD, predominantly hyperlipidemia and diabetes $(8,9)$.

Therapeutic strategies that exist in the prevention of TCAV are limited. Options for management of TCAV include immunosuppressive therapy, coronary allograft revascularization [percutaneous coronary intervention (PCIs), coronary artery bypass grafting (CABG)], or heart re-transplantation (10-12). Heart re-transplantation is the definitive management of TCAV in this group of patients, but is limited by the scarcity of suitable donor organs (13). Despite extensive literature regarding the use of PCI and CABG in the management of CAD, there is a paucity of data about the efficacy of such interventions in TCAV.

The primary objective of this systematic review was to examine the outcomes of PCI as compared to CABG in the management of TCAV. Our secondary objective was to compare the use and outcomes of drug eluting stents (DES) as compared to bare metal stents (BMS) among patients who underwent PCI for TCAV.

\section{Methods}

\section{Literature search strategy}

Thorough electronic searches were performed in August 2017 using Ovid Medline, Embase, Cochrane Central
Register of Controlled Trials (CCTR), Cochrane Database of Systematic Reviews (CDSR), Web of Science, Scopus and CINAHL. To achieve the maximum sensitivity of the search strategy, we combined the terms: "heart transplant", "cardiac transplant", "coronary artery disease", "myocardial infarction", "myocardial ischemia", "vasculopathy", "coronary stenosis", "revascularization", "angioplasty", "stent" and "coronary artery bypass" as either key words or MeSH terms. The reference lists of all retrieved articles were reviewed for further identification of potentially relevant studies, assessed using the inclusion and exclusion criteria.

\section{Selection criteria}

Eligible studies for the present systematic review and metaanalysis included those that addressed TCAV amongst heart transplant recipients. When institutions published duplicate studies with accumulating numbers of patients or increased lengths of follow-up, only the most complete reports were included for quantitative assessment with no overlapping time intervals. We excluded studies on patients $<18$ years of age, studies not published in the English language and those not involving human subjects. Furthermore, abstracts, case reports, conference presentations, editorials, reviews and expert opinions were also excluded.

\section{Data extraction and critical appraisal}

Data was extracted from article texts, tables and figures $\mathrm{JH}$ Choi, JG Luc). Discrepancies between the two reviewers were resolved by discussion and consensus.

\section{Statistical analysis}

A meta-analysis of proportions was conducted for the available main perioperative and postoperative variables with logit transformation. Heterogeneity was evaluated using Cochran $\mathrm{Q}$ and $\mathrm{I}^{2}$ test. Meta-regression was conducted using PCI vs. CABG as a subgrouping variable, or BMS vs. DES in case of our secondary objective. Studies included in the BMS $v s$. DES analysis did not include studies that report mixed techniques (BMS and DES combined) or those that do not specify stent type. R software, version 3.01 (R Foundation for Statistical Computing, Vienna, Austria) was used for all data analysis and visualization. The metaanalysis was performed using metafor package for Rusing continuity correction factor of 0.5 . P values $<0.05$ were considered statistically significant. 


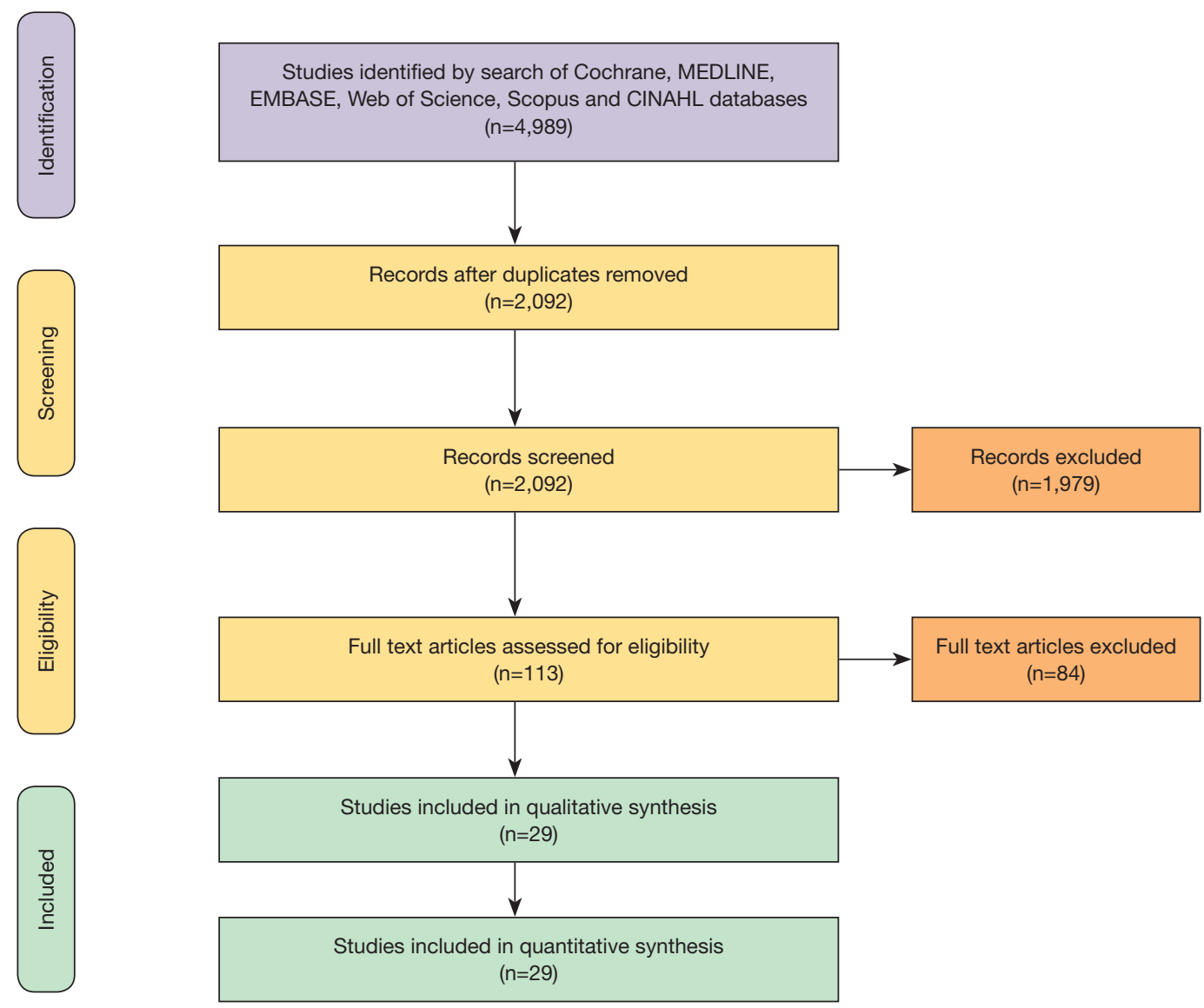

Figure 1 PRISMA schematic of search strategy. PRISMA, Preferred Reporting Items for Systematic Reviews and Meta-Analyses.

\section{Results}

\section{Study characteristics}

Overall, 4,989 records were identified in the literature search that were published between 1968 and 2015. Following application of the inclusion and exclusion criteria, 29 studies were included for analysis, with a total of 1,520 patients and mean follow-up period of 30.8 months. A PRISMA flow diagram depicting the overall search strategy is provided in Figure 1. These studies included 28 singlecenter studies, and 1 multicenter registry. Of the studies, 28 studies had retrospective patient enrolment and 1 study had prospective patient enrolment. Manual search of references did not yield further studies.

\section{Baseline demographics}

Baseline demographics are shown in Table 1. Mean patient age was 54.4 years ( $95 \%$ CI, 52.66-56.06) with $78.6 \%$ being male. Patient comorbidities include hypertension (80.0\%), diabetes $(35.8 \%)$, dyslipidemia $(69.5 \%)$, prior myocardial infarction (20.5\%) and obesity (53.3\%). Etiology of heart failure requiring heart transplantation was primarily ischemic cardiomyopathy (55.5\%).

In terms of initial clinical presentation of TCAV, the majority of patients $(55.4 \%)$ were asymptomatic with other variants of presentation including angina (24.8\%), acute coronary syndrome (22.4\%), congestive heart failure $(18.3 \%)$ and myocardial infarction (10.6\%) (Table 2). The majority of involved lesions were those of the left anterior descending coronary artery $(67.8 \%)$, followed by right (38.1\%), left circumflex $(32.6 \%)$ and left main coronary artery $(7.1 \%)$ (Table 2). Some patients had lesions in multiple areas.

\section{PCI vs. CABG for TCAV cohorts}

Among 1,520 patients who developed TCAV, 1,470 patients 


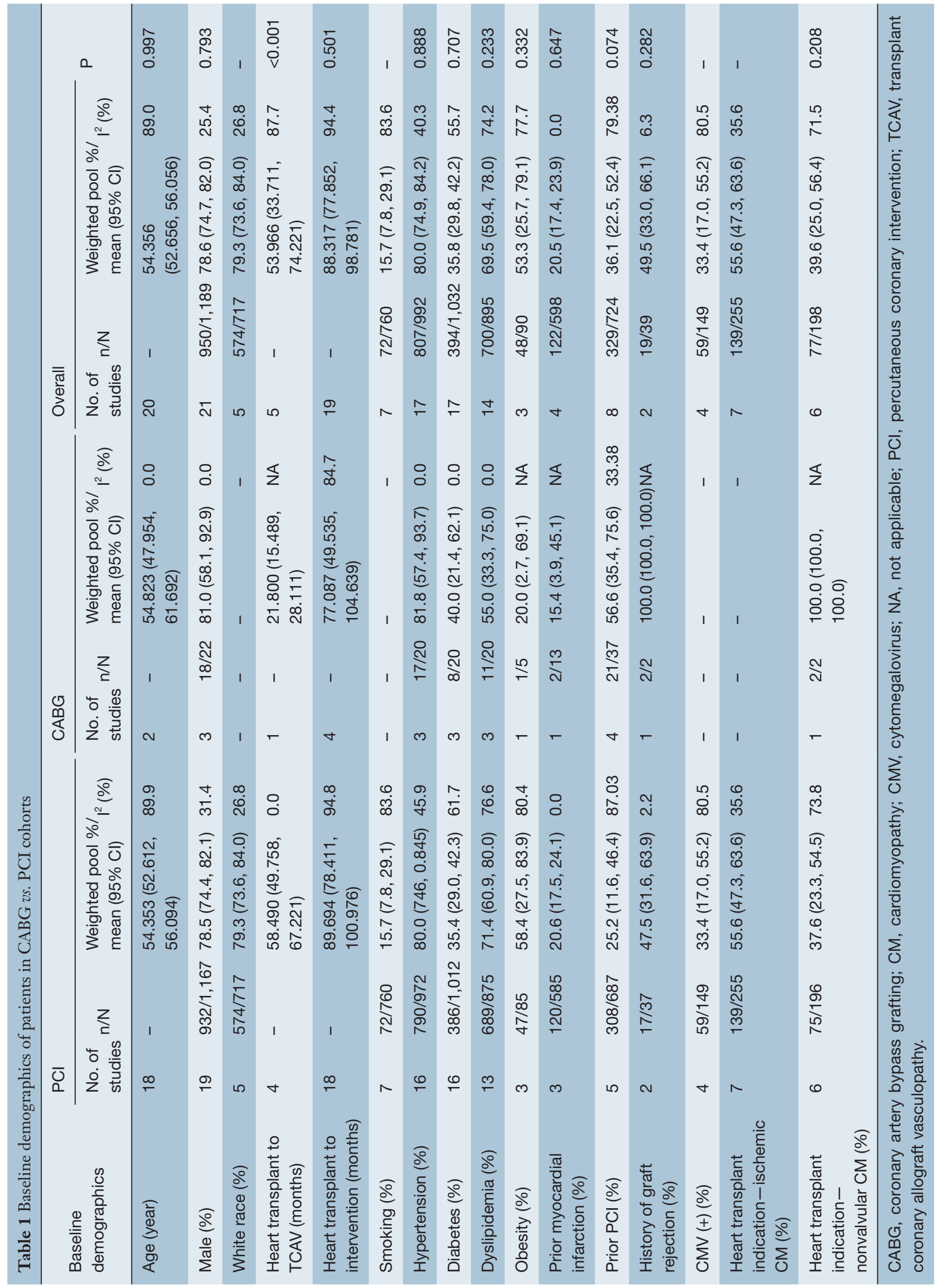




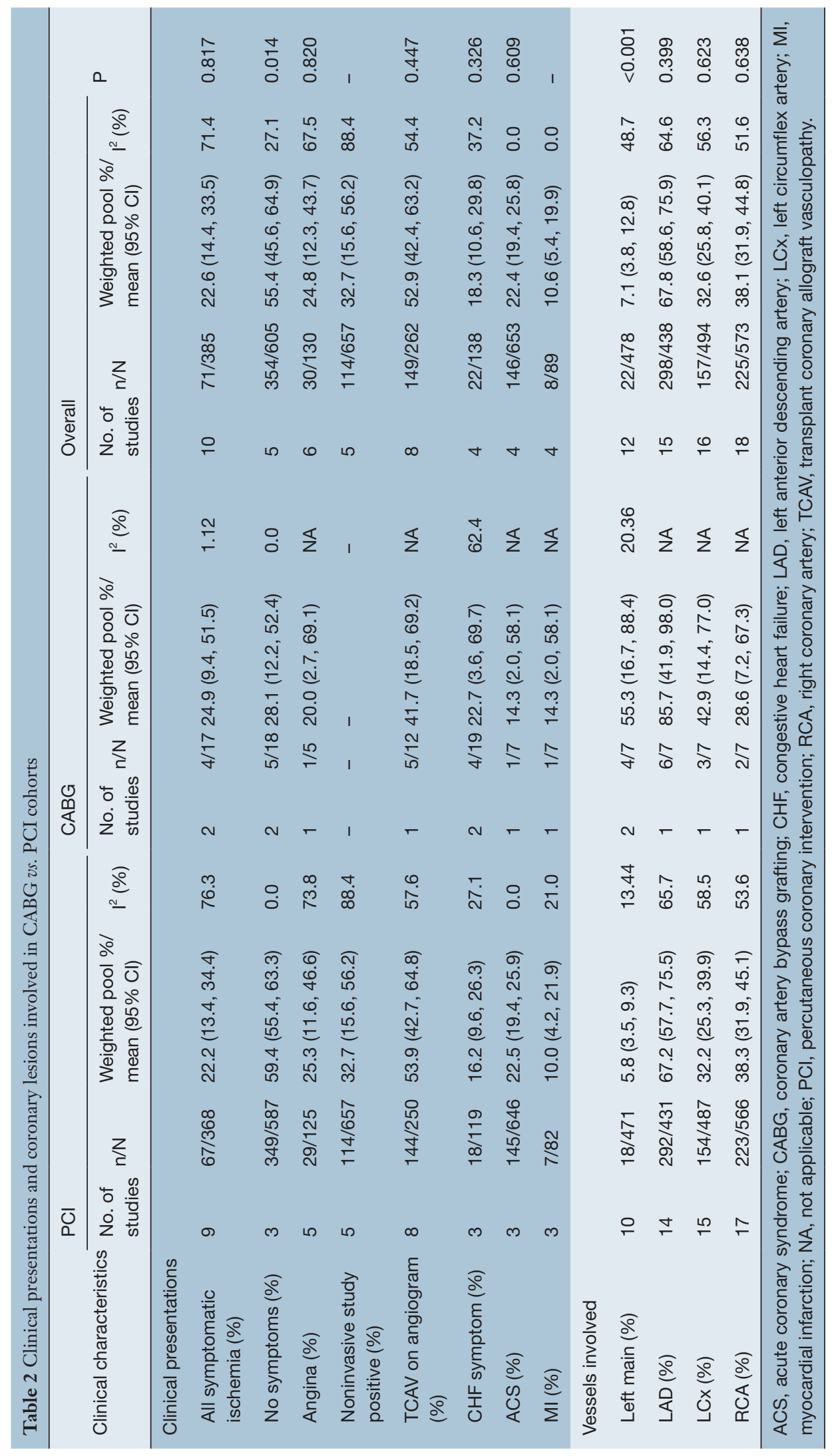


(96.7\%) underwent PCI and 50 patients (3.3\%) underwent CABG surgery. When comparing patients with TCAV who underwent PCI as compared to CABG, there were no significant differences in regards to mean age [PCI 54.4 (95\% CI, 52.61-56.09) vs. CABG 54.8 years (95\% CI, 47.95-61.69), $\mathrm{P}=0.997]$ or other comorbidities. Patients who underwent PCI were more likely to be farther out from their primary heart transplantation [PCI 58.49 (95\% CI, 49.76-67.22) vs. CABG 21.80 months (95\% CI, 15.50 28.11), $\mathrm{P}<0.001$ ] and asymptomatic [PCI 59.4\% (95\% CI, 55.4-63.3) vs. CABG 28.1\% (95\% CI, 12.2-52.4), $\mathrm{P}=0.014$ ] compared to the CABG cohort (Table 2).

Patients with left main TCAV were more likely to undergo CABG as compared to PCI [CABG 55.3\% (95\% CI, 16.7-88.4) vs. PCI 5.8\% (95\% CI, 3.5-9.3), $\mathrm{P}<0.001]$ (Table 2). There were no significant differences in the likelihood of patients undergoing $\mathrm{CABG}$ as compared to PCI for other coronary lesions, including the left anterior descending artery [CABG $85.7 \%$ (95\% CI, 41.9-98.0) vs. PCI $67.2 \%$ (95\% CI, 57.7-75.5), $\mathrm{P}=0.399$ ], left circumflex artery [CABG $42.9 \%$ (95\% CI, 14.4-77.0) vs. PCI $32.2 \%$ (95\% CI, 25.3-39.9), $\mathrm{P}=0.623$ ] or right coronary artery [CABG 28.6\% (95\% CI, 7.2-6.3) vs. PCI 38.3\% (95\% CI, 31.9-45.1), $\mathrm{P}=0.638$ ].

For the entire cohort, early mortality, as defined as mortality within 30 days of TCAV intervention or hospital discharge, was $13.5 \%$ (95\% CI, 5.0-31.6). Compared to patients who underwent PCI, those who underwent CABG had a higher early mortality [CABG 36.4\% (95\% CI, 20.0-56.7) vs. PCI 4.3\% (95\% CI, 2.1-8.8), $\mathrm{P}<0.001]$ and overall mortality [CABG $42.3 \%$ (95\% CI, 28.4-57.5) vs. PCI $21.4 \%$ (95\% CI, 14.4-30.7), $\mathrm{P}=0.049$ ], but no difference in cardiac-related mortality [CABG 32.5\% (95\% CI, 19.7-48.5), PCI $22.7 \%$ (95\% CI, 15.1-32.7), $\mathrm{P}=0.362$ ]. CABG was accompanied with a trend towards decreased need for repeat intervention [CABG 15.4\% (95\% CI, 3.9-45.1) vs. PCI $37.2 \%$ (95\% CI, 16.1-64.7), $\mathrm{P}=0.327]$ that did not reach statistical significance $(\mathrm{P}=0.327)$ during the same follow-up period [CABG 22.41 (95\% CI, 24.259-39.388) vs. PCI 31.82 months (95\% CI, 0-51.640), $\mathrm{P}=0.337$ ] (Table 3).

\section{Drug eluting versus BMS}

Among the 303 patients who underwent PCI in studies reporting on stent type, 181 patients received DES (60\%) and 122 patients received BMS (40\%). Preoperative medications received among the cohorts are described in
Table 4. Compared to the BMS cohort, patients in the DES cohort were more likely to receive the following medications in addition to aspirin: clopidogrel [DES $81.7 \%$ (95\% CI, 56.1-94.0) vs. BMS 36.8\% (95\% CI, 18.7-59.7), $\mathrm{P}=0.004]$, angiotensin converting enzyme inhibitors/angiotensin receptor blockers [DES 56.4\% (95\% CI, 42.1-69.7) vs. BMS 33.1\% (95\% CI, 21.8-46.7), $\mathrm{P}=0.007$ ], statins [DES 80.6\% (95\% CI, 73.1-86.4) vs. BMS 69.2\% (95\% CI, 56.8 79.3), $\mathrm{P}=0.077$ ] and oral sirolimus [DES $43.5 \%(95 \% \mathrm{CI}$, 27.8-60.7) vs. BMS 21.6\% (95\% CI, 11.2-37.7), $\mathrm{P}=0.045]$. Conversely, patients receiving BMS were more likely to receive oral azathioprine [BMS 53.1\% (95\% CI, 20.5-83.3) vs. DES 15.0\% (95\% CI, 8.5-25.2), $\mathrm{P}=0.014]$. No differences in immunosuppressive therapy were seen between the DES and BMS cohort in regards to administration of cyclosporine, mycophenolate, or tacrolimus.

In terms of periprocedural details (Table 5), those who received a BMS were more likely to have a higher postprocedure minimal lumen diameter [BMS 3.075 (95\% CI, 2.752-3.398) vs. DES 2.571 mm (95\% CI, 2.433-2.708), $\mathrm{P}<0.001$ ], stent diameter [BMS 3.197 (95\% CI, 3.155-3.239) vs. DES $3.000 \mathrm{~mm}$ (95\% CI, 2.963-3.037), $\mathrm{P}<0.001]$ and length [BMS 20.295 (95\% CI, 18.284-22.307) vs. DES $18.740 \mathrm{~mm}$ (95\% CI, 17.161-20.319), $\mathrm{P}<0.001]$.

There were no significant differences in the rate of instent stenosis [DES 14.5\% (95\% CI, 4.5-38.0) vs. BMS 24.4\% (95\% CI, 16.9-33.9), $\mathrm{P}=0.476$ ], overall mortality [DES 17.4\% (95\% CI, 9.0-31.0) vs. BMS 30.8\% (95\% CI, 12.0-59.1), $\mathrm{P}=0.302$ ] or cardiac related mortality [DES 7.7\% (95\% CI, 2.9-18.8) vs. BMS 21.8\% (95\% CI, 10.4-40.2), $\mathrm{P}=0.415$ ] (Table 5). Furthermore, there were no significant differences between the DES and BMS cohorts in terms of survival (Figure 2A), event-free survival (Figure 2B) and freedom from restenosis (Figure 2C) at 1,2 and 3 years post PCI.

\section{Discussion}

Coronary allograft vasculopathy following heart transplantation has an incidence of $30-50 \%$ at 5 years (14) and remains the most common cause of late allograft failure and the major determinant for long-term survival (3). The mainstays of treatment include CABG, PCI or heart retransplantation (15). Although the definitive management of TCAV is retransplantation, it is limited by the scarcity of suitable donor organs. Therefore, retransplantation is usually reserved for patients with disease progression despite revascularization or those with diffuse coronary 

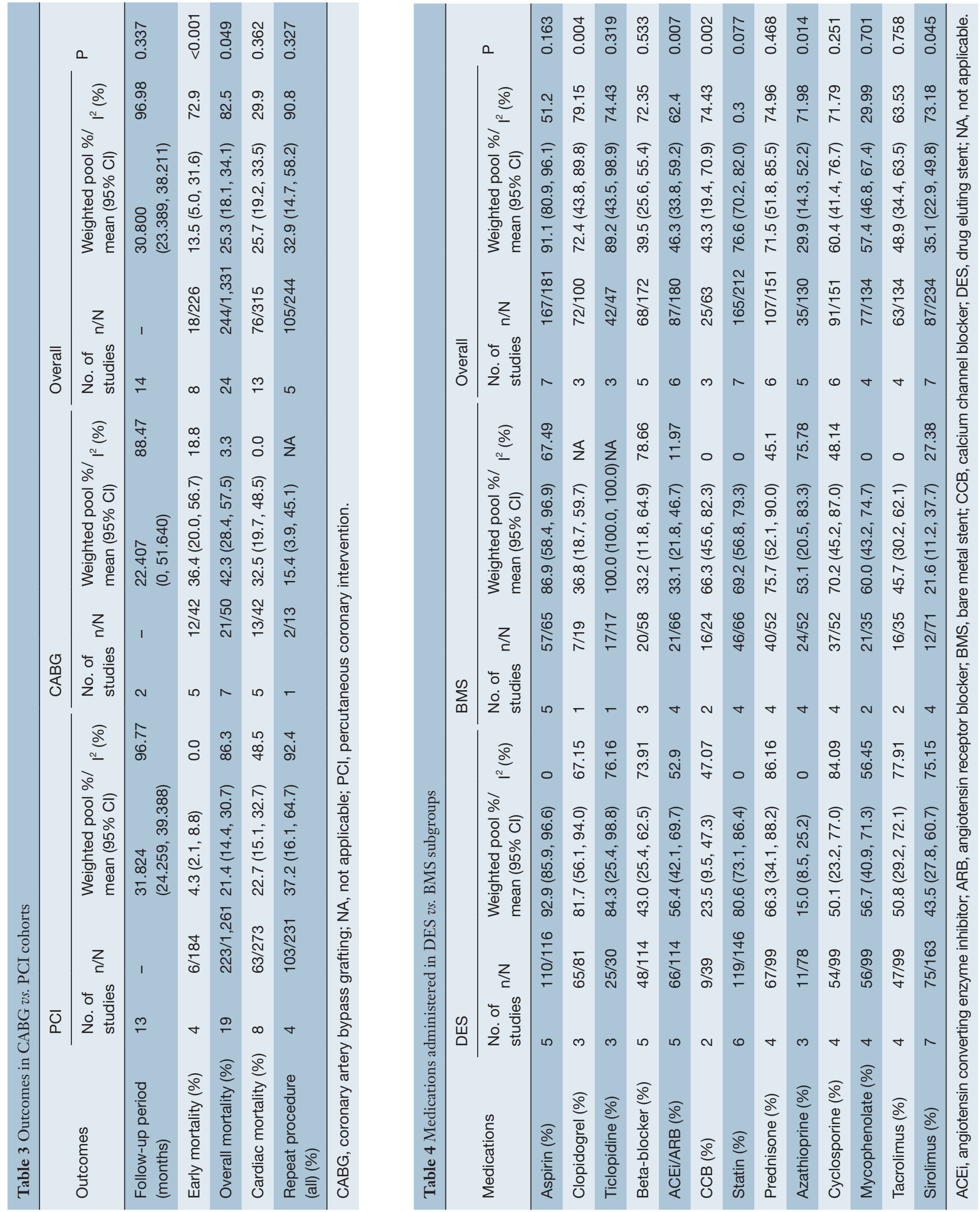


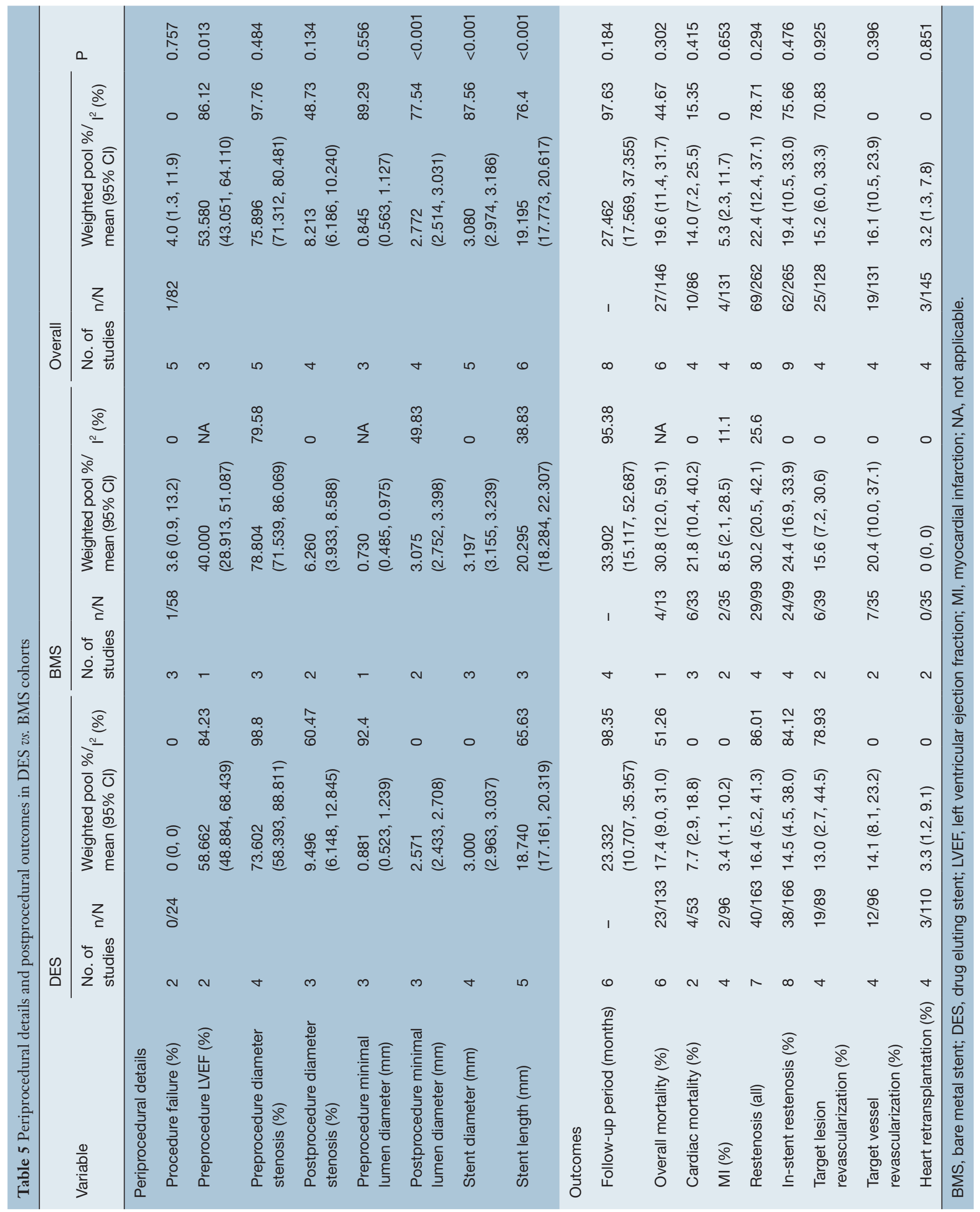



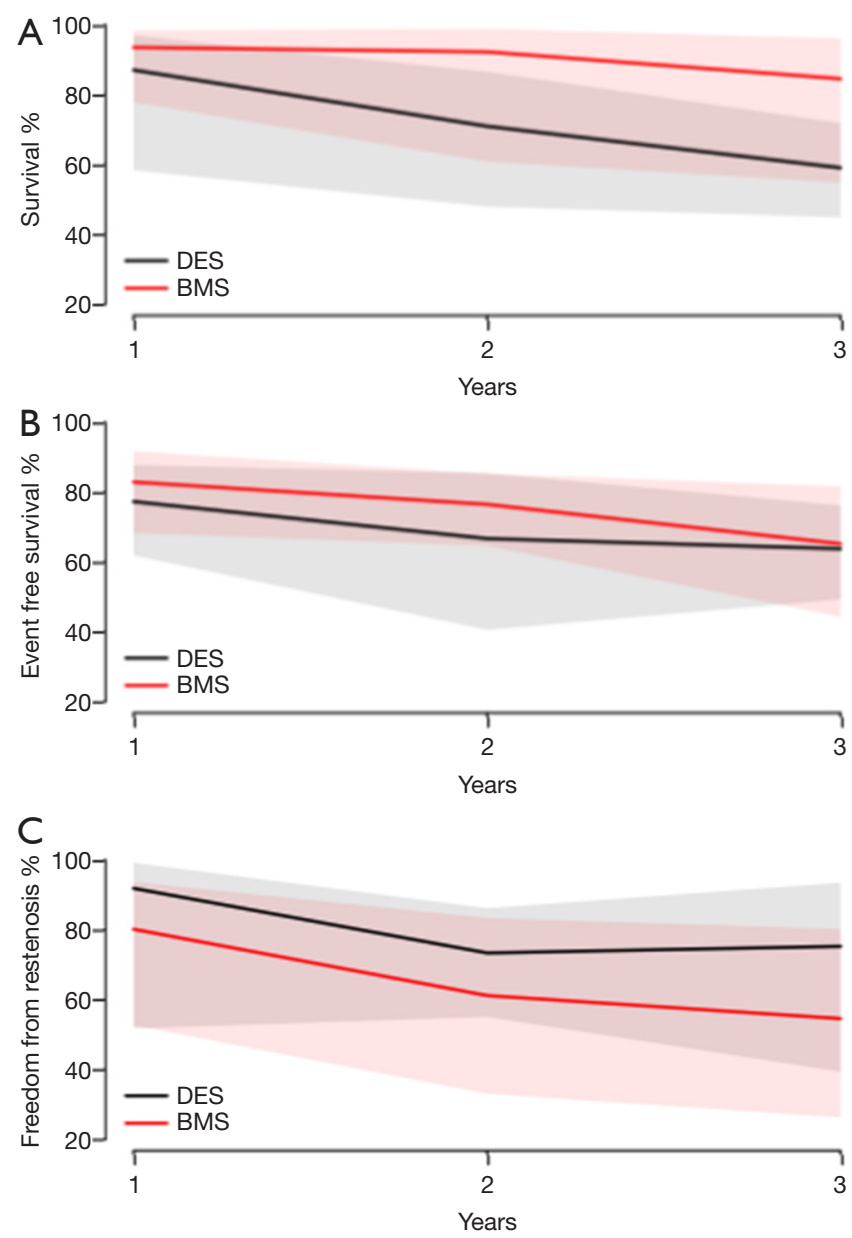

Figure 2 Comparison between DES and BMS in terms of (A) survival, (B) event-free survival and (C) freedom from restenosis at 1, 2 and 3 years of follow-up. DES, drug eluting stents; BMS, bare metal stents.

involvement (13). Limited studies have compared the outcomes of CABG and PCI in the management of TCAV.

In this systematic review and meta-analysis, we demonstrate that both CABG and PCI are feasible modalities for revascularization in patients with TCAV, where PCI is associated with lower mortality and no difference in cardiac mortality during the same follow up period. Furthermore, among those who underwent PCI, there were no differences in outcomes in terms of mortality, event-free survival or freedom from restenosis for patients who received a DES compared to a BMS.

In contrast to $\mathrm{CAD}$ in the general population, TCAV has significant morphological differences and risk factors (4), and has been thought to be a manifestation of chronic

rejection (16). Preventative strategies for TCAV include treatment of immunological (anti-HLA, chronic inflammation and acute rejection via optimization of immunosuppressive therapy) and nonimmunological factors (e.g., hypertension, hyperlipidemia and pre-existing diabetes) (17). Early post-transplant coronary angiography is often obtained in recipients of higher risk donor hearts to screen for the presence of pre-transplant coronary artery disease as there has been shown a correlation between the presence of pre-transplant coronary artery disease and the incidence and severity of TCAV (18).

We demonstrate that CABG in patients with TCAV was associated with higher mortality $(\mathrm{P}<0.001)$, though no significant difference was found in cardiac-related mortality $(\mathrm{P}=0.362)$. Furthermore compared to $\mathrm{PCI}, \mathrm{CABG}$ in patients with TCAV was accompanied with a decreased need for repeat intervention $15.4 \%$ vs. $37.2 \%$ that did not reach statistical significance $(\mathrm{P}=0.327)$ during the same follow-up period. Given the aforementioned outcomes, it is vital to interpret the results with care, whilst keeping in mind the threshold for revascularization in this often asymptomatic group of patients. There is significant heterogeneity in CABG early mortality outcomes for patients with TCAV $(10,14,18,19)$, which is likely due to the limited number of patients in each study, differences in patient selection, treatment bias, and changes in medical management and surgical practice across the decades. Furthermore, in the present study, average time from heart transplantation to revascularization was shorter $(\mathrm{P}<0.001)$ with more patients with TCAV involving the left main coronary artery in the CABG subgroup as compared to the PCI group $(\mathrm{P}<0.001)$. As such, despite a higher incidence of TCAV with left main involvement in the CABG cohort, CABG cannot be advocated readily and indiscriminately given the significant early morbidity and mortality.

Prior decision algorithms have suggested that CABG can be performed in the subgroup of heart transplant patients with Type A lesions, whereby Type B/C lesions are not amenable to bypass surgery (20). However, the decision for CABG surgery should depend on other factors as well, factors including adequate artery size (minimum of 1.8 to $2 \mathrm{~mm}$ ), whether the combination of lesions at different stages are bypassable, and whether the lesions are hemodynamically significant (14). Other factors that warrant consideration in patient selection include the patient's overall physiological and functional status, comorbidities, likelihood of achieving complete revascularization, and whether alternative surgical approaches such as off-pump 
techniques are applicable (14).

PCI in patients with TCAV has been associated with greater restenosis rates compared to PCI in patients with native CAD (21). As DES has been shown to reduce restenosis when implanted in native coronary arteries (22-24), we compared the use and outcomes of DES vs. BMS among patients who underwent PCI for TCAV. In the present study, there was a non-significant higher freedom from restenosis at 1,2 and 3 years among those who received a DES as compared to a BMS with no significant difference in mortality. Existing reports share a common experience $(25,26)$. Similarly, a meta-analysis of six different studies found no difference in terms of major adverse cardiac events (MACE) or all-cause mortality between DES vs. BMS (27). Reasons postulated as to why DES did not affect MACE or all-cause mortality is that TCAV is a diffuse and progressive process, that a focal lesion is a marker of widespread disease (28), and that repeat intervention will remain common regardless of method of PCI.

Due to the denervation of the afferent cardiac nervous system and absence or incomplete reinnervation of the cardiac allograft, most heart transplant patients present asymptomatically, and if at all, with atypical angina (20), as is evidenced in our current study. Therefore, the first clinical manifestations of coronary allograft ischemia predominantly include congestive heart failure, ventricular arrhythmias, silent myocardial infarction, or sudden cardiac death (29). As such, given its asymptomatic nature and progressive nature of TCAV, close and frequent surveillance for heart transplant recipients is necessary. Noninvasive studies such as the treadmill test or myocardial perfusion studies have been demonstrated to have limited clinical utility due to poor sensitivity for TCAV, owing to the diffuse nature of the disease which does not allow for assessment of differential myocardial blood flow on radionuclide scans (16). Dobutamine echocardiographic stress test has been shown to be a more sensitive noninvasive screening test than the former noninvasive tests, nonetheless angiography remains the current gold-stand in diagnosis and characterization of lesions (16). The incremental levels of sensitivity from noninvasive to invasive tests for TCAV are demonstrated in the current study, where the majority of TCAV were found on angiography. However, angiography has been shown to underestimate the presence and severity of TCAV (20). Intravascular ultrasound imaging (IVUS) has been proposed as a more sensitive means of detecting intimal vascular wall thickening and may be an important tool for ensuring optimal stent deployment, reducing stent under-expansion, incomplete stent apposition, edge dissection or geographic miss (30).

\section{Limitations}

This meta-analysis has several key limitations and must be interpreted with care. Regional differences exist in patient and donor selection, center experience, heart transplantation techniques, immunosuppressive regimes used and clinical management of heart failure. We acknowledge that this heterogeneity in study population is a fundamental limitation that cannot be addressed due to inability to extract sufficient detail from the pooled data. Due to the lack of detail in the data, we were unable to stratify CABG outcomes by era or analyze and compare multi-lesion interventions separately. Pooled results may not correctly reflect the advancements made during the last five decades of this widely performed procedure. $\mathrm{n} / \mathrm{N}$ variation amongst variables reported led to fluctuation in pooled number results. Moreover, the heterogeneity in results precludes broad generalization into prognostic terms. The impact of CABG and PCI in patients with TCAV on survival and freedom from restenosis is difficult to assess due to the limited number of patients, lack of detail precluding the ability to differentiate patients who underwent complete $v s$. partial revascularization, and incomplete clinical and angiographic follow-up for some of the patients. Furthermore, inconsistent definitions were used to describe the outcomes and CABG and PCI in terms of restenosis and clinically significant coronary lesions, with inconsistent criteria for the need for repeat reintervention. It is possible that patients undergoing PCI represented a population that was less sick than the population that underwent CABG, thereby overestimating the benefit of PCI. As well, patients who underwent CABG as compared to PCI may be different and represent different states in the natural course of progression of TCAV. It is also plausible that outcomes of revascularization may have been influenced by clinical presentation rather than treatment modality.

In view of improving success of cardiac transplantation, a larger number of patients with TCAV can be anticipated to present in need of therapy. It is hoped that advances in noninvasive methods to improve screening for TCAV using either imaging studies (31) or noninvasive markers (32) continue to expand for earlier diagnosis. Furthermore, ongoing research in TCAV and vascular biology to develop targeted preventive therapies, interventional and surgical 
therapeutic approaches are needed to tailor management and improve outcomes. Decisions regarding CABG vs. PCI are likely to continue to remain difficult and it is our hope that the present study forms the basis for future studies as well as provides a platform for dialogue and collaboration amongst multiple disciplines to work towards reducing the number of grafts lost to TCAV. Randomized controlled trials or prospective registry analysis with clinical and angiographic data comparing outcomes of CABG vs. PCI for patients with TCAV would be invaluable.

\section{Conclusions}

The results of our systematic review of 29 studies consisting of 1,520 patients with TCAV demonstrate that CABG and PCI are both feasible modalities for revascularization in patients with TCAV, with PCI being associated with lower mortality. There were no differences in outcomes among patients who underwent PCI with DES as compared to BMS. Further studies are needed to delineate evidencebased guidelines to tailor the appropriate therapy, CABG or PCI, to the appropriate patient with TCAV.

\section{Acknowledgements}

None.

\section{Footnote}

Conflicts of Interest: The authors have no conflicts of interest to declare.

\section{References}

1. Yusen RD, Edwards LB, Kucheryavaya AY, et al. The Registry of the International Society for Heart and Lung Transplantation: thirty-second official adult lung and heart-lung transplantation report-2015; Focus Theme: Early Graft Failure. J Heart Lung Transplant 2015;34:1264-77.

2. Lund LH, Edwards LB, Dipchand AI, et al. The Registry of the International Society for Heart and Lung Transplantation : Thirty-third Adult Heart Transplantation Report - 2016 ; focus theme : primary diagnostic indications for transplant. J Heart Lung Transplant 2016;35:1158-69.

3. Lund LH, Edwards LB, Kucheryavaya AY, et al. The registry of the International Society for Heart and Lung
Transplantation: thirty-first official adult heart transplant report--2014; focus theme: retransplantation. J Heart Lung Transplant 2014;33:996-1008.

4. Ramzy D, Rao V, Brahm J, et al. Cardiac allograft vasculopathy: a review. Can J Surg 2005;48:319-27.

5. Mehra MR, Crespo-Leiro MG, Dipchand A, et al. International Society for Heart and Lung Transplantation working formulation of a standardized nomenclature for cardiac allograft vasculopathy-2010. J Heart Lung Transplant 2010;29:717-27.

6. Hussain T, Burch M, Fenton MJ, et al. Positive pretransplantation cytomegalovirus serology is a risk factor for cardiac allograft vasculopathy in children. Circulation 2007;115:1798-805.

7. Szyguła-Jurkiewicz B, Szczurek W, Gąsior M, et al. Risk factors of cardiac allograft vasculopathy. Kardiochir Torakochirurgia Pol 2015;12:328-33.

8. Kemna MS, Valantine HA, Hunt SA, et al. Metabolic risk factors for atherosclerosis in heart transplant recipients. Am Heart J 1994;128:68-72.

9. Kato T, Chan MCY, Gao SZ, et al. Glucose intolerance, as reflected by hemoglobin A1c level, is associated with the incidence and severity of transplant coronary artery disease. J Am Coll Cardiol 2004;43:1034-41.

10. Halle AA, DiSciascio G, Massin EK, et al. Coronary angioplasty, atherectomy and bypass surgery in cardiac transplant recipients. J Am Coll Cardiol 1995;26:120-8.

11. Kuroda K, Sunami H, Matsumoto Y, et al. Percutaneous coronary intervention and coronary artery bypass grafting in heart transplant recipients with transplant coronary arterial vasculopathy. Transplant Proc 2017;49:130-4.

12. Agarwal S, Parashar A, Kapadia SR, et al. Long-term mortality after cardiac allograft vasculopathy: implications of percutaneous intervention. JACC Heart Fail 2014;2:281-8

13. Radovancevic B, McGiffin DC, Kobashigawa JA, et al. Retransplantation in 7,290 primary transplant patients: A 10-year multi-institutional study. J Heart Lung Transplant 2003;22:862-8.

14. Bhama JK, Nguyen DQ, Scolieri S, et al. Surgical revascularization for cardiac allograft vasculopathy: Is it still an option? J Thorac Cardiovasc Surg 2009;137:1488-92.

15. Prada-Delgado O, Estévez-Loureiro R, López-Sainz A, et al. Percutaneous coronary interventions and bypass surgery in patients with cardiac allograft vasculopathy: a single-center experience. Transplant Proc 2012;44:2657-9.

16. Sharifi M, Siraj Y, O'Donnell J, et al. Coronary angioplasty and stenting in orthotopic heart transplants: a fruitful act 
or a futile attempt? Angiology 2000;51:809-15.

17. Costanzo MR, Naftel DC, Pritzker MR, et al.

Heart transplant coronary artery disease detected by coronary angiography: a multiinstitutional study of preoperative donor and recipient risk factors. Cardiac Transplant Research Database. J Heart Lung Transplant 1998;17:744-53.

18. Parry A, Roberts M, Parameshwar J, et al. The management of post-cardiac transplantation coronary artery disease. Eur J Cardiothorac Surg 1996;10:528-32; discussion 53.

19. Musci M, Loebe M, Wellnhofer E, et al. Coronary angioplasty, bypass surgery, and retransplantation in cardiac transplant patients with graft coronary disease. Thorac Cardiovasc Surg 1998;46:268-74.

20. Musci M, Pasic M, Meyer R, et al. Coronary artery bypass grafting after orthotopic heart transplantation. Eur J Cardiothorac Surg 1999;16:163-8.

21. Zimmer RJ, Lee MS. Transplant coronary artery disease. JACC: Cardiovasc Interv 2010;3:367-77.

22. Morice MC, Serruys PW, Sousa JE, et al. A randomized comparison of a sirolimus-eluting stent with a standard stent for coronary Revascularization. N Engl J Med 2002;346:1773-80.

23. Moses JW, Leon MB, Popma JJ, et al. Sirolimus-eluting stents versus standard stents in patients with stenosis in a native coronary artery. N Engl J Med 2003;349:1315-23.

24. Stone GW, Ellis SG, Cox DA, et al. One-year clinical results with the slow-release, polymer-based, paclitaxeleluting TAXUS stent: the TAXUS-IV trial. Circulation 2004;109:1942-7.

25. Bader FM, Kfoury AG, Gilbert EM, et al. Percutaneous coronary interventions with stents in cardiac transplant

Cite this article as: Luc JG, Choi JH, Rizvi SA, Phan K, Moncho Escrivà E, Patel S, Reeves GR, Boyle AJ, Entwistle JW, Morris RJ, Massey HT, Tchantchaleishvili V. Percutaneous coronary intervention versus coronary artery bypass grafting in heart transplant recipients with coronary allograft vasculopathy: a systematic review and meta-analysis of 1,520 patients. Ann Cardiothorac Surg 2018;7(1):19-30. doi: 10.21037/ acs.2018.01.10 recipients. J Heart Lung Transplant 2006;25:298-301.

26. Tarantini G, Favaretto E, Gardin A, et al. Drug-eluting stents for the treatment of coronary lesions in cardiac transplant vasculopathy: acute and mid-term clinical and angiographic outcomes. J Cardiovasc Med (Hagerstown) 2008;9:396-402.

27. Dasari TW, Hennebry TA, Hanna EB, et al. Drug eluting versus bare metal stents in cardiac allograft vasculopathy: a systematic review of literature. Catheter Cardiovasc Interv 2011;77:962-9.

28. Simpson L, Lee EK, Hott BJ, et al. Long-term results of angioplasty vs stenting in cardiac transplant recipients with allograft vasculopathy. J Heart Lung Transplant 2005;24:1211-7.

29. Marelli M, Rábago G, Calabuig J, et al. Midterm results of percutaneous transluminal coronary angioplasty in graft coronary artery disease in cardiac transplant patients. Transplant Proc 1999;31:2539-41.

30. Madder RD, VanOosterhout S, Klungle D, et al. Multimodality intracoronary imaging with near-infrared spectroscopy and intravascular ultrasound in asymptomatic individuals with high Calcium ScoresCLINICAL PERSPECTIVE. Circ Cardiovasc Imaging 2017;10:e006282.

31. Gregory SA, Ferencik M, Achenbach S, et al. Comparison of sixty-four-slice multidetector computed tomographic coronary angiography to coronary angiography with intravascular ultrasound for the detection of transplant vasculopathy. Am J Cardiol 2006;98:877-84.

32. Mehra MR, Uber PA, Potluri S, et al. Usefulness of an elevated B-type natriuretic peptide to predict allograft failure, cardiac allograft vasculopathy, and survival after heart transplantation. Am J Cardiol 2004;94:454-8. 